\title{
Situations AND THe Liar PARAdoxes
}

\author{
Guilherme ARAúJo CARDoso \\ Centro Federal de Educação Tecnológica de Minas Gerais; Centro de Lógica, Epistemologia \\ e História da Ciência (Unicamp); CNPq, BRAzIL \\ guilhermeprimeiro@gmail.com
}

\begin{abstract}
In this paper we intend to outline an introduction to Situation Theory as an approach to the liar paradoxes. This idea was first presented by the work of Barwise and Etchemendy (in their (1987)). First (section 1) we introduce the paradoxes in their most appealing and important versions. Second (section 2) we show that non-classical approaches on the problem usually get puzzled by the revenge problem on one side and loss of expressive power on the other side. Last (sections 3 and 4), we present Situation Theory and try to show how it is capable of solving the old paradoxes and blocking revenge. The price we pay on this view is universality, since it would allow a new revenge situated liar. We don't intend to address the problem of universality here, but we try at least to motivate the reader to make sense of this theory.
\end{abstract}

Keywords: $\quad$ Liar paradoxes $\bullet$ revenge problem $\bullet$ bivalence $\bullet$ T-schema $\bullet$ universality $\bullet$ nonwell-founded propositions $\bullet$ situation theory

\section{Liar Paradoxes}

For a fast and straight statement of the main versions of the liar's family, just consider the following list of funny sentences:

(1) (1) is false.

(2) (2) is not true. ${ }^{1}$

(3) (4) is true.

(4) (3) is not true.

(5) (5) is not true and Donald Trump is the President of USA.

(6) If (6) is true, everything is true.

(7) Consider the next infinite list, where $x$ ranges over natural numbers:

$\left(7_{1}\right)$ For all $x>1,\left(7_{x}\right)$ is not true.

$\left(7_{2}\right)$ For all $x>2,\left(7_{x}\right)$ is not true. 
$\left(7_{3}\right)$ For all $x>3,\left(7_{x}\right)$ is not true.

$\left(7_{m}\right)$ For all $x>m,\left(7_{x}\right)$ is not true.

$\left(7_{m+1}\right)$ For all $x>m+1,\left(7_{x}\right)$ is not true.

The common fact about the sentences above is that they all generate paradoxes. In a broader sense, a paradox is an argument apparently valid in which all premisses look true but its conclusion looks false. Now, if we take for premisses the Principle of Bivalence and T- Schema, we can show (by valid arguments ${ }^{2}$ ) that each one of the aforementioned sentences is true iff it is not true, from where we can also obtain an infinite list of contradictions. We are about to show this. Before that, let us talk about the premisses of the arguments. The first premisse is the Principle of Bivalence: ${ }^{3}$

Bivalence: Let $\mathscr{L}$ be a classically interpreted first order language and let $p$ be any proposition expressed by a sentence of $\mathscr{L}$. Hence, either $p$ is true or $p$ is not true (it is false), and not both.

We also assume that natural (ordinary) concept of truth ${ }^{4}$ is a classical one. Hence, if sentences (1)-(7) (taking (7) for the infinite list given by it) express propositions, each one of them is either true or false and none of them is both true and false. The classical interpretation of negation together with Bivalence also implies that, for each proposition $p, p$ is false iff $\neg p$ is true. Now we introduce second premisse, that means, the T-Schema:

T-Schema: Let $\mathscr{L}$ be a classically interpreted and semantically closed first order language and let $p$ be any proposition expressed by a sentence of $\mathscr{L}$. Hence,

$$
\operatorname{Tr}(p) \text { is true iff } p \text { is true. }{ }^{5}
$$

Generally speaking, we assume that natural language is capable of expressing its very own semantic (it is a semantically closed language). In particular, it assumes that natural language can talk about what is expressed by its very own sentences. As a consequence, natural language has its very own truth predicate and this predicate must mirror transparency given by T-Schema. Any predicate $A(x)$ of $\mathscr{L}$ that doesn't verify T-Schema cannot be taken as a truth predicate of $\mathscr{L}$. The main reason for this is that T-Schema is very basic.

We are now left with the question concerning the existence of propositions expressed by sentences (1)-(7). When dealing with sentences, this is obviously unnecessary. Those sentences are all proved to exist in any interpreted language that verifies the antecedent of Diagonal Lemma. Self-reference, cross-reference and infinite 
sequences of references are usually taken to be formalized by this lemma. ${ }^{6}$ Nevertheless, here we need to assume something stronger: we need to assume that beyond the existence of those sentences, the propositions expressed by them also exist. Therefore, we need to agree with the existence of non-well-founded propositions. This an important notion we borrow from sets. Let's first define what is a non-well-founded relation on sets.

Well-founded relations: A relation $R$ is well-founded on a set $X$ iff, for any subset $Y$ of $X$, there is a $R$-minimal element $y$ in $Y$, that is, there is a $y \in Y$ such that, for no $z \in Y, z R y .^{7}$

Consider now the relation of reference on sentences. ${ }^{8}$ From above definition, we see that reference is a non-well-founded relation on any set of sentences that includes (1)-(7). Hence, the propositions expressed by these sentences would be called nonwell-founded propositions. Later, we'll be representing propositions by sets. Hence, non-well-founded propositions shall be represented by non-well-founded sets.

Our third premisse is the assumption that sentences (1)-(7) really express propositions, that means, we assume that there are non-well-founded propositions. It seems to be a very intuitive assumption concerning natural language, but if the reader is looking for additional reasons to accept the existence of non-well-founded propositions, consider the examples given by the following sentences:

(8) The proposition expressed by (8) is an example of non-well-founded propositions.

(9) Everything expressed in this paper could also be expressed in Portuguese.

We won't argue for the authenticity of the propositions expressed by (8) and (9). But if we agree that 'true' applies to propositions, it would be just wrong to say that (8) and (9) cannot express propositions, because they both seem to express something true in the current context.

Let's now run the paradoxes. For simplicity, we are going to take sentences (1)(7) as the propositions expressed by them. By footnote 2, we can just ignore version (1). Thus, (2) is the proposition that says of (2) it is not true, hence (2) $=\neg \operatorname{Tr}(2)$. By T-Schema, (2) is true iff $\operatorname{Tr}(2)$ is true. For the last identity, however, (2) is true iff $\neg \operatorname{Tr}(2)$ is true. Therefore, $\operatorname{Tr}(2)$ is true iff $\neg \operatorname{Tr}(2)$ is true and, by T-Schema again, (2) is true iff $\neg(2)$ is true. This also implies (from classical negation) that (2) is true iff (2) is not true. Finally, from Bivalence, (2) is true and (2) is not true. Similar reasoning follows from the fact that $(3)=\operatorname{Tr}(4)$ and $(4)=\neg \operatorname{Tr}(3)$. The paradox given by (3) and (4) is usually known as the Postal Card's Paradox.

Consider now the proposition $\left(7_{m}\right)$, where $m$ is any positive integer. By Bivalence, we know that $\left(7_{m}\right)$ is either true or not true (and not both). On the other side, from 
what it says, $\left(7_{m}\right)$ is true iff $\left(7_{m+1}\right)$ is not true, $\left(7_{m+2}\right)$ is not true, $\left(7_{m+3}\right)$ is not true, and so on (for all $\left(7_{x}\right)$, where $x>m$ ). ${ }^{9}$ But that is not possible. Suppose $\left(7_{n}\right.$ ) (for any $n>m$ ) is not true. Hence, for some $x>n,\left(7_{x}\right)$ is true, making $\left(7_{m}\right)$ not true and then contradicting hypothesis. Therefore, $\left(7_{m}\right)$ cannot be true. Suppose that it is not true. Now, for some $x>m,\left(7_{x}\right)$ is true, but that is not possible, as we have just seen for any positive integer $m$. Accordingly, for each proposition expressed by the infinite list of sentences given by (7), this proposition is true iff it is false (not true). This version of the liar is usually known as Yablo's Paradox. ${ }^{10}$ There is a discussion on whether Yablo's sentences result from circularity or not. Despite that, we can easily see here that the propositions expressed by them are non-well-founded ones and that is enough.

Proposition (5) is a special kind of problem. Well, it is a fact of the world where we are (during the year of 2017) that Donald Trump is the President of USA. Therefore, if we give 'and' its classical interpretation, then (5) is true iff (5) is not true. Nevertheless, sentence (5) doesn't have to produce a paradox, because it could be the case that Trump is not the President of USA. Trump could lose the elections, or sentence (5) could just express a different proposition if asserted in the year of 2012. In that case, we could just say that (5) is not true (or false) and this is not a paradox, because it doesn't imply that (5) is true. This nice version of the liar was designed by Kripke (1975) as an argument to show that the liars can result from contingent facts and extrinsic features of the sentences. For this reason, these versions of the liar are usually known as Contingent Liars.

Contingent liars play with complex propositions and classical interpretations of sentential operators. However, we can use the same pieces for different paradoxical results, those exemplified by (6). Suppose (6) is not true. Thus, from classical interpretation of 'if ..., then ...', the antecedent of (6) must be true and its consequent must be false. But this isn't possible, because the antecedent of (6) is $\operatorname{Tr}(6)$. If (6) is not true, (6) is true. Suppose then that (6) is true. Hence, antecedent of (6) is also true and, by Modus Ponens, everything is true. This is called Curry's Paradox. It is a paradox that seems to derive triviality from above premisses despite any contradictions. In Classical Logic, we have this principle that derives triviality from contradictions, the so called Principle of Explosion. Of course, from triviality we could derive contradictions (we could derive anything whatsoever). Nevertheless, here we could derive triviality directly from (6). Notice that if we change the consequent of (6) for something contingently false, we would be running contingent liars once again.

These are the liars. Let's now see a very brief sketch of the main intended approaches on them and their general problems. We intend to show that these approaches on the liar are challenged by a very hard puzzle between deep limits of expressive power from one side and the Revenge Problem on the other side. 


\section{Expressive Power and The Revenge Problem}

In the last section we tried to show that liar paradoxes come from three basic premisses plus Classical Logic: Bivalence, T-Schema and non-well-founded propositions. At first glance, we could find reasons to deny some of these premisses. Denying Bivalence is the road taken by the so called Non-Classical Views of the liar. Of course, depending on what you change in classical interpretations, T-Schema is also affected. We could also find reasons to deny T-Schema or the very existence of non-wellfounded propositions inside hierarchies. This road is the one taken by Hierarchical Views. Consider a hierarchy of truth predicates, for instance. In this case, there is not only a single truth predicate (there are many of them), making T-Schema ambiguous in a sistematic way. ${ }^{11}$ We can also consider something like a hierarchy of meaning domains over which propositions can properly be about, making non-well-founded propositions nonsense. ${ }^{12}$

The main objection against hierarchies is that they usually lose expressive power. Consider for instance that we split 'true' in an infinite number of indexed predicates under a hierarchy of levels. There is an implicit index $m$ for the predicate $\operatorname{Tr}$ in (2) so that (2) itself cannot be in the extension nor in the anti-extension of $T r_{m}$, but (2) has to be either in the extension or the anti-extension of $T r_{m+1}$. In this setting, (2) cannot be used to express a proposition that says of itself it is not true. The paradox is blocked. However, we would like the ordinary concept of truth to be taken by a single predicate (no relative implicit levels). When Bivalence is taken for granted about natural language, we want to say for instance that the following sentence expresses a true proposition:

(10) For each proposition $p$, either $p$ is true or $p$ is not true (and not both).

If we accept the hierarchy of $\operatorname{Tr}$ levels, we can not take (10) to express a true proposition. In such a hierarchy, if (10) expresses a proposition, then there is a level $m$ implicit in the occurrences of $\operatorname{Tr}$ in (10) so that (10) itself needs to be in a higher level $n$ (for some $n>m$ ). Hence, there is at least one proposition that is not in either the extension or in the anti-extension of $\operatorname{Tr}_{m}$, making (10) just wrong, more precisely, making (10) a false $_{n}$ proposition. Hence, it looks that we cannot express semantical facts about all propositions in such a hierarchical perspective, unless we allow this hierarchy to have some kind of fixed point, a special level $\omega$ of $\operatorname{Tr}$ in which (10) is a non-well-founded true proposition. ${ }^{13}$ Let $\operatorname{Tr}_{\omega}$ be such a predicate that ranges over any proposition. Now we can say that (10) is right, actually it is true $\omega_{\omega}$, but what happens then with the next sentence?

(11) (11) is not true ${ }_{\omega}$. 
(11) is a revenge version of the liar, a version that comes from an ineffective solution. We need a fixed point of the hierarchy so that we can have expressive power, but then we fall puzzled by liars again. Therefore, it looks that hierarchical views are facing a hard dilemma: to lose the alleged expressive power of natural language or to accept a revenge version of the liar. We intend to show next that something similar happens with the non-classical views.

We understand non-classical views as those that deny bivalence. Of course, denying bivalence can mean at least two different things: denying the exhaustive part of it (made explicit by the expression 'or' in (10)) or denying its exclusive part (made explicit by the expression 'not both' in (10)). The first alternative is called Gap View, for the reason that it allows some propositions to be neither true nor false, falling in a kind of gap between true and false. The second alternative is called Glut View, because it allows some propositions to be both true and false: it has truth values in a glut. We shall be showing next that none of these two alternatives seem to work very well.

The paradigmatic example of the Gap View on the liar is Kripke's theory. ${ }^{14}$ However, Kripke takes sentences as truth bearers. Some sentences just do not express propositions and fall in the gap of truth values. The reason for this is that Kripke is trying to give a model that allows us to formalize the differences between the grounded sentences, the ungrounded sentences and the paradoxical sentences. ${ }^{15}$ For Kripke, sentences (1)-(7) above don't express propositions, so they can not be evaluated as either true or false. The problem with those sentences however is not a syntactical one, they all are well formed declarative sentences that could be used in different contexts to express meaningful propositions. Nevertheless, something outside them is wrong, something inside the empirical circumstances or the contexts in which they have been stated here. It's like claiming in the year of 2017 that the present king of France is bald. We could express something either true or false with this in a different context, but today it would just make no sense to say something like that. ${ }^{16}$

Suppose we accept that (2) is neither true nor false. Suppose that (2) falls in a gap of truth values ((2) doesn't have a truth value) or it has a different third value. Let's say, in both cases, that (2) is gappy. From the fact that (2) is gappy doesn't follow that (2) is true and also doesn't follow that (2) is false. Therefore, we could consistently say that (2) is gappy, making room for a non-hierarchical solution to the liar. Nevertheless, this solution seems to open ways for revenge. Consider the next sentence:

(12) (12) is gappy or (12) is false.

(12) is a special kind of liar. Like those from the beginning ((1) to (7)), (12) can not be consistently evaluated as true or false. If it is true, then it is either gappy or 
false. If it is false, then it is true (its second part is true). However, (12) can not be gappy either, because that would make (12) true (the first part of it would be true). We have a paradox once again.

In Kripke's models we can not run (12), because the predicate 'gappy' is not available. There is a language $\mathscr{L}$ in which the predicate $\operatorname{Tr}(x)$ represents the set of true sentences of $\mathscr{L}$ and a partial interpretation $\mathfrak{A}$ so that paradoxical sentences like (1) to (7) are not in the extension nor in the anti-extension of $\operatorname{Tr}$. But we are not forced to add predicate 'gappy' in $\mathscr{L}$. Of course, outside of $\mathscr{L}$ we can see that (2) is gappy, but this is not enough for running a revenge paradox in $\mathscr{L}$. Nevertheless, there is a problem here. The main reason why we chose to accept a non-classical interpretation is that we believed that it could allow us to solve the liars with no expressive damages like those of hierarchical views. Even so, we have just seen that we could not express in $\mathscr{L}$ this very important fact that (2) is gappy, otherwise revenge looks inevitable. Therefore, the main lesson we should take from (12) is that the Gap View needs to make a very hard choice: expressive power or consistency. If the language can express the fact that (2) is gappy, (12) can be obtained in it. If, otherwise, gappy is not available in it, (12) is also not available, but now we can not express in this very same language that (2) is gappy.

Consider now the very radical and extreme perspective taken by the Glut Views. According to these, sentences like (1) to (7) express propositions that are both true and false, they express contradictions. This is the route taken, for instance, by dialetheists. ${ }^{17}$ We are not going to discuss here the nature of contradictions. In what follows, we only show some objections to the Glut Views that do not depend on such matters.

The Glut Views seem to keep expressive power safe and they also seem to block revenge versions of the liar. How could a revenge argument be used to show that Glut View is wrong, if all it can do is to show the existence of new contradictions? Are the Glut Views then free from revenge problems? We don't think so. The revenge in this case can't be used to show inconsistency, but triviality, which is the new ghost, the one capable of scaring even those that challenge consistency.

It can be defended that one of the main reasons why we take contradictions to be false is that, if they were true, we would have to accept anything whatsoever, we would have triviality. To allow triviality is like giving up any sort of logical constraint. In Classical Logic, we have Principle of Explosion, which allows us to prove anything from a single true contradiction. Thus, if we accept that there are true contradictions, we need to block general validity of Explosion. This is exactly what Glut Views usually do, by adopting alternative logics. Nevertheless, we can show by at least two arguments that Glut Views can derive triviality by reasons that seem to be independent of the general validity of Explosion. These arguments can then be presented as revenge versions of the liar for the Glut Views. 
The first argument is just Curry's Paradox exemplified by sentence (6), once again. Of course, Glut Views can say that (6) is both true and false. The problem here, however, is clearly not solved by this. If (6) is both true and false, then (a fortiori) (6) is true, but if that is the case, (by Modus Ponens) everything is true. The paradox is about triviality, not about consistency. This problem can be obviously solved by changing the way we interpret conditionals, but the consequences of this kind of change in the expressive power of such language would not be so easy to live with.

This is an old, very well known and discussed point. Dialetheism has been already, from the beginning, conceived in a way to block this kind of problem. The way Priest presents Dialetheism, it doesn't preserve Modus Ponens nor take conditional as the one given by classical interpretations. Therefore, we don't have Curry's Paradox. Nevertheles, we could easily think of Modus Ponens as a natural and valid rule of inference, one that mirrors very well the conditionals of ordinary languages. Furthermore, we can derive triviality from Glut Views by another version of revenge, as we are going to see now.

When a given proposition $p$ is true, we can also say that the $\{$ True $\}$ is the set of values of $p$. The same thing works for the value False. However, this would imply that if $p$ is both true and false, the set of values of $p$ is $\{$ True, False $\}$. At first glance, nothing is wrong here, but if the language we are taking into account can express such facts about sets of values of its own propositions, a new form of revenge is available, as can be exemplified by the next sentence:

(13) The set of values of (13) is $\{$ False $\}$.

(13) is obviously a version of liar. It can't be consistently evaluated as true or false. What happens then if we take (13) to be both true and false, as suggested by Glut Views? In that case, a fortiori, (13) is true, from where we can infer that the set of values of (13) is $\{$ False (by T-Schema). At the same time, if (13) is both true and false, the set of values of (13) is $\{$ True, False $\}$. Therefore, $\{$ False $\}=\{$ True, False . By Extensionality, ${ }^{18}$ we conclude that True $=$ False , and we get triviality once again .

Thus, we conclude that both Hierarchical Views and Non-Classical Views are faced with the same very hard dilemma, the one between loss on the expressive power of theories and the revenge versions of the old paradoxes. In this setting, the ideal solution would certainly be one capable of preserving good expressive power, but without revenge.

\section{Situation Theory}

There is an alternative to Hierarchical and Non-Classical views, it is the Contextualist View. The basic idea behind this view is that contexts play an indispensable 
role in expressing propositions. For each sentence $A$ and context $s_{n}$, there is only one proposition $p$ such that $p$ is the proposition expressed by $A$ relative to $s_{n}$. Hence, the reason why we can't consistently think of truth values on liar sentences like (2) is that it can express different propositions relative to different contexts. In particular, (2) can express a true proposition relative to a given context $n$ and it can express a false proposition relative to another context $m$. Therefore, by taking sentences as truth bearers, we hide context ambiguities and get paradoxes as results. Otherwise, each proposition (which includes a sentence relative to a given context) can be consistently evaluated as true or false and no paradoxes would arise. The basic idea behind contextualism is nice, but it can be used by very different theories. ${ }^{19}$ In this paper we focus attention on one version of Contextualist View, the Situation Theory of Barwise and Etchemendy, which we believe to fit the aforementioned requirements for a good solution of the liar paradoxes. Let's see how it works.

From an informal perspective, we can think of situations as proper parts of the world, where each part is detached by the insertion of an informational agent into a given space-time location. Thus, situations are determined by agents, space-time locations and worlds. ${ }^{20}$ Of course, different space-time locations for the same agent in the same world can give rise to different situations, as much as different agents in the same space-time locations of the same world give rise to different situations.

Consider, for instance, the morning of November 5 during the year 2015 in Bento Rodrigues, Brazil. At that point, Antônio Quintão was playing with his ninety birds and he thought that it was a beautiful day. This is a situation. ${ }^{21}$ The described situation verifies a bunch of facts (states of affairs), like the fact that there were birds singing in the yard of Antônio Quintão, the fact that he thought it was a beautiful day and the fact that there was a Brazilian sub-district named Bento Rodrigues. A very distinct situation could be taken by the perspective of one of those birds, a different informational agent. Both agents are differently attuned to the same space-time locations, they are differently capable of extracting information from it, giving rise to different situations. Another distinct situation was about to happen later during the same day, when a huge dam broke, flooding the whole sub-district and causing the worst environmental disaster in Brazil's history. In this new situation, there were no more singing birds, actually almost the whole sub-district of Bento Rodrigues was now changed from the perspective of Antônio Quintão. The slice of the world seen by Quintão in the morning is different from the slice of the world he sees at night as much as it differs from the the slice of the world seen by birds at the same morning. Nevertheless, all those slices belong to the same world, they are situations of the same world.

This is how situations are related with worlds and agents. Let's leave aside any concerns about the concept of attunement (that relates agents to worlds). ${ }^{22}$ In what follows, we give formal definitions of the main concepts used above whitin the frame- 
work of set theory. We need a set theory that not only accepts non-well-founded sets, but actually takes their existence for granted. In Barwise and Etchemendy (1987), the set theory is $Z F C^{-} / A F A$, that means, ZFC less Foundation Axiom plus the AntiFoundation Axiom. While Foundation Axiom excludes the existence of non-wellfounded sets in ZFC, AFA guarantees the existence of these sets. The way AFA does that can not be explained here, but the reader should find a good explanation in the third chapter of Barwise and Etchemendy (1987).

Definition 1. A State of Affair (or SOA) is a $n+2$-tuple like $\left(R, o_{1}, \ldots, o_{n}, i\right)$, where $R$ is a n-place relation, $o_{1}, \ldots, o_{n}$ are objects ${ }^{23}$ and $i$ is an element of the set $\{1,0\}$.

When two SOA's are only distinguished by the element $i$ inside each one of them, like $\left(R, o_{1}, \ldots, o_{n}, 1\right)$ and $\left(R, o_{1}, \ldots, o_{0}, 0\right)$, they are usually called duals related to each other. The element 1 marks the positive SOA and the element 0 marks the negative SOA. We can also represent the negative SOA by $\left(\overline{R, o_{1}, \ldots, o_{n}, 1}\right)$.

Definition 2. A situation is any set of SOA's.

Suppose we have a situation that includes both a given SOA $\sigma$ and its dual $\bar{\sigma}$. This would make the situation an inconsistent class. Well, this is not the kind of situation that we believe to be detachable from the actual world we live in. Actually, we would like to say that these situations aren't even possible ones. Of course, we don't have arguments against the idea that the world could include inconsistent classes, but at this point we are already looking for alternatives to Dialetheism. Therefore, this is just an assumption.

Some situations can be possible even if they verify SOA's that are not facts in our world. These are some of the main reasons why we need to distinguish between only possible situations and actual situations. Instead of talking about worlds, however, we are going to talk about models of the world.

Definition 3. A partial model of the world $\mathfrak{A}$ is any class of SOA's satisfying the following conditions:

(i) $\mathfrak{A}$ is a consistent class of SOA's, that means, either $\sigma \notin \mathfrak{A}$ or $\bar{\sigma} \notin \mathfrak{A}$ (for any $S O A$ $\sigma)$.

(ii) If $(\operatorname{Tr}, p, 1) \in \mathfrak{A}$, then $p$ is true.

(iii) If $(T r, p, 0) \in \mathfrak{A}$, then $p$ is false. ${ }^{24}$

The reader should probably have noticed that conditions ii and iii of definition 3 are very closely related with one of the two directions of T-Schema. Let's call it the downward direction of T-Schema. The idea here is that a partial and actual model of the world should be such that if it is a fact of this world that proposition $p$ is true 
(false), then proposition $p$ must be really true (false). Of course, we are not talking about languages in this setting, but just relating propositions with the world. Later, we are going to show that total models of the world also imply the upward direction of T-Schema. Then we should be able to talk about semantically closed models of the world, which is not the same thing as semantically closed languages, but something really similar and closely related to it.

Definition 4. A situation $s$ is actual in the model $\mathfrak{A}$ iff $\mathfrak{A}$ contains $s . s$ is possible iff it is actual in some model.

Strictly speaking, the notion of truth would be relative to models. However, we are taking the actual world (the actual model of the world) as fixed, but an actual model of the world can still be a total one or not. This is why we could still have different actual models of the same world. Next definition concerns totality of the models of worlds.

Definition 5. A total model of the world is any partial model of the world such that it is not properly contained in a partial model of the world.

Let's make a brief pause for some intermediate results. First consider that situations can be partial in a way that is closely related to gaps. Suppose, for instance, that Linda is in a situation $s$ such that she can not see that Claire has the A maybe because there is no Claire in the space-time locations where Linda is, maybe because Linda can't see the cards of Claire in this game. Hence, (Has, Claire, A, 1$) \notin s$ and (Has, Claire, $A \mathbf{\$}, 0) \notin s$. Of course, it is a fact of this world that Claire does have the $A \boldsymbol{\phi}$, but situation $s$ is not one that carries this information. Even if $s$ is an actual situation, it doesn't mean that $s$ informs the agent of the whole world. A total model of the world can't share this partiality of situations. In fact, we can prove the following:

Theorem 6. Let $\mathfrak{A}$ be a total model. Thus either $\sigma$ is in $\mathfrak{A}$ or $\bar{\sigma}$ is in $\mathfrak{A}$, and not both (for any SOA $\sigma$ ).

Proof. We prove it by reductio ad absurdum. Suppose that $\mathfrak{A}$ is a total model and, for some SOA $\sigma, \sigma \notin \mathfrak{A}$ and $\bar{\sigma} \notin \mathfrak{A}$. Thus, there is a model $\mathfrak{A}^{\prime}=\mathfrak{A} \cup\{\sigma\}$ such that $\mathfrak{A}$ is properly contained in $\mathfrak{A}^{\prime}$, which contradicts the initial hypotesis of $\mathfrak{A}$ being a total model. Therefore, either $\sigma \in \mathfrak{A}$ or $\bar{\sigma} \in \mathfrak{A}$. Suppose, otherwise, that both $\sigma$ and $\bar{\sigma}$ are in $\mathfrak{A}$. In this case, $\mathfrak{A}$ is an inconsistent class of SOA's, therefore it is not a model, because of condition $i$ in definition 3 .

We have already defined situations and models of the world, the first as sets of SOA's and the second as classes of SOA's that preserve consistency and downward direction of T-Schema. The use of the words 'classes' and 'sets' here, as the reader 
can guess, is not an arbitrary one. In fact, we can show that total models can not be sets (they need to be proper classes), what a fortiori also implies that no situation can be a total model of the world. This is an important result for the main point of this paper, therefore it deserves being highlighted as a theorem.

Theorem 7. There is no situation $s_{u}$ and total model $\mathfrak{A}$ such that $s_{u}=\mathfrak{A}$.

Proof. Let $s_{u}$ be a situation and $\mathfrak{A}$ be a total model. First we show that $\mathfrak{A}$ can not be a set. From definition 2, it will follow then that $\mathfrak{A}$ is not a situation, therefore, $s_{u} \neq \mathfrak{A}$. From theorem 6 , it follows that $\mathfrak{A}$ includes at least one SOA for each class $A$, either (Finite, $A, 1$ ) or (Finite, $A, 0$ ), for instance. ${ }^{25}$ This makes $\mathfrak{A}$ too inclusive, since it includes all sets. For this reason, $\mathfrak{A}$ can not be a set. ${ }^{26}$

An interesting point related with theorem 7 deserves being mentioned. This theorem excludes the existence of a universal and absolute situation, a situation that would be equal to the actual and total model of whole world. As we mentioned before, a situation is always determined by an agent in a given space-time location. Hence, theorem 7 implies that there is no agent and space-time location that could determine a situation equal to a total model of the world, that means, there is no universal agent attuned to the whole world (no omniscient agent, no universal perspective). This result could be taken as an additional reason for theorem 7 , since it correlates the theorem with very plausible limitations on real agents and real spacetime locations.

We have already defined situations and models of worlds. Now we need to talk about the bearers of truth, propositions. As already mentioned, we are going to take propositions as structured entities represented by sets. We also want to capture the contextualist idea that context always plays some role on determining the propositions expressed by sentences. In Barwise and Etchemendy (1987), these two important features of propositions are grasped by the Austinian perspective, according to which propositions are always about situations. In fact, a proposition can be determined by the very situation which it is about and by the type it says that such situation is.

Let's first understand the notion of Situation Type. We can see that situations share some SOA's with others. For instance, consider the situation $s^{\prime}$ in which Max has the $A \boldsymbol{\phi}$ and the situation $s^{\prime \prime}$ in which Claire has the $A \boldsymbol{\phi}$. In this case, $s^{\prime}$ and $s^{\prime \prime}$ have in commom the fact that someone has an $A$. We can say that every situation $s$ in which someone has the $A \boldsymbol{s}$ is of the same type of $s^{\prime}$ and $s^{\prime \prime}$. A type could be so taken as a class of situations determined by some given SOA $\sigma$, that means, the type of $\sigma$ is the class of all situations that verify $\sigma$. We represent the type of situations determined by $\sigma$ with $[\sigma]$. For technical reasons, $[\sigma]$ can not define the class of all situations $s$ such that $\sigma \in s .{ }^{27}$ We just assume it to be the ordered pair $(\sigma, 1)$. 
This way we can still distinguish the SOA $\sigma$ from the situation type determined by it, $[\sigma]$.

A type can be also determined in a complex way, by more than just one SOA. For instance, we can talk about the type in which either $\sigma$ or $\tau$ verify. We can also talk about the type in which both SOA's verify. Consider a given set of types $X$. From $X$ we can also obtain two more types: i) [ $\bigwedge X]$, the type of all situations $s$ such that $\sigma \in s$, for all $[\sigma] \in X$. ii) $[\bigvee X]$, the type of all situations $s$ such that $\sigma \in s$, for some $[\sigma] \in X$.

We can now introduce propositions and next define truth:

Definition 8. A proposition $p$ is any set $p=\{s, T\}$ such that $s$ is a situation and $T$ is a situation type.

As we said before, the proposition $p=\{s, T\}$ is the one that says of situation $s$ that it is of type $T$. Definition 8 makes really easy to grasp what it is for a proposition to be true.

Definition 9. Let $s$ be a situation, $\sigma$ a SOA and $X$ a set of types. Thus:

(i) $p=\{s,[\sigma]\}$ is true iff $\sigma \in s$.

(ii) $p=\{s,[\bar{\sigma}]\}$ is true iff $\bar{\sigma} \in s$.

(iii) $p=\{s,[\bigwedge X]\}$ is true iff $\{s, T\}$ is true, for each $T \in X$.

(iv) $p=\{s,[\bigvee X]\}$ is true iff $\{s, T\}$ is true, for some $T \in X$.

Consider for instance the proposition $p=\{s,[\bigvee\{[\sigma],[\tau]\}]\}$, where we assume that $\sigma=($ Has, Claire,A, 1$)$ and $\tau=($ Has, Max,A, 1$)$. The proposition $p$ says of situation $s$ that it verifies either the fact that Claire has the $A$ or the fact that Max has the A. Notice that $p$ could be true even if none of these two SOA's are facts of the real world, because $s$ doesn't have to be an actual situation. Moreover, $p$ could be false even if both SOA's are facts of the real world, because even if $s$ is actual, it doesn't follow by this that it verifies all facts of the real world. Nevertheless, we can still prove Bivalence:

Theorem 10. For any proposition $p$, either $p$ is true or $p$ is false, and not both.

Proof. The proof runs by induction on complexity of types inside definition 9. Consider first clause i of definition 9, that means, there is a situation $s$ and a SOA $\sigma$ such that $p=\{s,[\sigma]\}$. From set theory, we have that $\sigma \in s$ or $\sigma \notin s$ and it's never the case that both happens. Therefore, $p$ is either true or false, and not both. The same thing holds when $p=\{s,[\bar{\sigma}]\}$. Consider now that $p=\{s,[\bigwedge X]\}$, according to clause iii of 9 , and $X$ is a set of types. Now, because we have already proved bivalence for 
propositions according to $\mathrm{i}$ and ii, we can presuppose that Bivalence works for each $q=\{s, T\}$, where $T \in X$. If all of them are true, then $p$ is true. Otherwise, $p$ is false. Hence, again, $p$ is either true or false, not both. Finally, when $p=\{s,[\bigvee X]\}$, we can also presuppose Bivalence for each $q=\{s, T\}$, where $T \in X$. If at least one of them is true, then $p$ is true. Otherwise, $p$ is false. Again, $p$ is either true or false, not both.

Bivalence asks for a brief comment on duality. Consider that the dual of a proposition $p=\{s, T\}$ is the proposition that says of $s$ it is of the dual type $\bar{T}$, that means, $\bar{p}=\{s, \bar{T}\}$. The reader should probably guess then that Bivalence is not about the relation between a proposition and its dual. In fact, it is possible that a proposition and its dual are both false, even if it is always the case that such proposition is either true or false (and not both). Consider, for instance, $p=\{s,[\sigma]\}$, where $\sigma=($ Has, Claire, $A \mathbf{\phi}, 1)$. It might be the case where $s$ doesn't verify $\sigma$ nor $\bar{\sigma}$. Maybe there is no Claire in situation $s$, whatsoever. Hence, both $p$ and $\bar{p}$ are false. How can we then express the related proposition that Bivalence establishes for each proposition? This can be done by adding the notion of denial. While the dual of a proposition is taken to be its negation, there is always a different proposition that stands for its denial:

Definition 11. Let $p=\{s, T\}$ be a proposition. Thus:

(i) Negation: $\bar{p}=\{s, \bar{T}\}$ is true iff $s$ is of type $\bar{T}$.

(ii) Denial: $\neg p$ is true iff $p$ is false.

Notice that Bivalence doesn't imply that, for each proposition and its negation, at least one is true, but it certainly implies that, for each proposition and its denial, at least one (exactly one) is true.

Another very important result that we can prove here, shows that total models can satisfy something similar to T-Schema. In a certain way, we can say that total models are semantically closed, which is one of the main desiderata for a good solution to the liar paradoxes. Let's see it.

Theorem 12. Let $\mathfrak{A}$ be a total model and let $p$ be any proposition. Thus:

(i) $(\operatorname{Tr}, p, 1)$ is in $\mathfrak{A}$ iff $p$ is true.

(ii) $(\operatorname{Tr}, p, 0)$ is in $\mathfrak{A}$ iff $p$ is false.

Proof. The left to right directions of $i$ and ii (the downward directions) follow directly from definition of partial models (definition 3 ). Suppose (for reductio) now that $p$ is true and $(T r, p, 1)$ is not in $\mathfrak{A}$. From $6,(T r, p, 0)$ is in $\mathfrak{A}$, and hence (by definition of partial models, again), $p$ is false, contradicting Bivalence (theorem 10). Therefore, if $p$ is true, $(\operatorname{Tr}, p, 1))$ is in $\mathfrak{A}$. The same reasoning works for proving ii. 
This is it. We have outlined Situation Theory. We expect that in this very brief outline the reader can grasp an idea of the great expressive power of the theory. It can preserve Bivalence and T-Schema, because total models are semantically closed. ${ }^{28} \mathrm{At}$ the same time, because of the partiality of situations, the theory can actually solve the liars, without leaving open the way for revenge. In the next section, we shall see how that is possible.

\section{The Liars in Situations}

We have already agreed to accept the existence of non-well-founded propositions. This seems enough for accepting a proposition that says of itself it is not true, but we are taking propositions to be about situations here, so we won't have just a single proposition that expresses the simple liar. In fact, we have an infinite number of simple liar propositions, depending on for which situation $s$ we instantiate the following schema:

$$
l=\{s,[(\operatorname{Tr}, l, 0)]\}
$$

Thus, there is something like a systematical indeterminacy inside the propositional form of the liars. For each situation $s_{x}$, there is a proposition $l_{x}=\left\{s_{x},[(\operatorname{Tr}\right.$, $\left.\left.\left.l_{x}, 0\right)\right]\right\}$ such that $l_{x}$ says of situation $s_{x}$ that it is of the type in which $l_{x}$ is not true. Does that make any difference? As we shall see next, it makes a huge difference the situation of which liar proposition is about. For the purpose of classification, consider at least two classes of situations the liar propositions can be about, the actual and the only possible (non actual) ones. This gives rise to two classes of simple liars: the accessible liars and the inaccessible liars. ${ }^{29}$

There is still more indeterminacy to expose. As we saw in last section, negation can be understood by two different perspectives, asserted negation or denial. Of course, this also makes important differences on representing the liars. The first schema of simple liars introduced above is for the asserted liars. Consider now the schema for denied liars:

$$
d=\neg\{s,[(T r, d, 1)]\}
$$

When we instantiate $s$ with some given situation, we have a proposition that denies of such situation that it is one of the type in which the proposition itself is true. Again, this situation can be actual or not. Therefore, we can have at least four classes of simple liars: i) asserted accessible liars, ii) asserted inaccessible liars, iii) denied accessible liars and iv) denied inaccessible liars. As we are about to see, they can all be classically and consistently evaluated as true or false.

Theorem 13. Asserted accessible liars are false propositions. 
Proof. Let $l_{1}=\left\{s_{1},\left[\left(\operatorname{Tr}, l_{1}, 0\right)\right]\right\}$, where $s_{1}$ is an actual situation. Suppose (for reductio) that $l_{1}$ is true. Thus (by truth definition) $\left(\operatorname{Tr}, l_{1}, 0\right) \in s_{1}$, and so $\left(\operatorname{Tr}, l_{1}, 0\right)$ is in $\mathfrak{A}$ (a total model), because $\mathfrak{A}$ includes $s_{1}$. But now 12 would imply that $l_{1}$ is false, contradicting Bivalence. By reductio, $l_{1}$ can not be true. Therefore (from Bivalence, again), $l_{1}$ is false.

Notice now that accepting that $l_{1}$ is false doesn't give rise to paradoxes. This actually implies that $\left(\operatorname{Tr}, l_{1}, 0\right)$ is in the total model $\mathfrak{A}$, but that doesn't matter, because $s_{1} \neq \mathfrak{A}$. Situation $s_{1}$ is an actual one, so it is part of total model $\mathfrak{A}$, but only as a proper part of it. Otherwise, we would have paradox again, because if $\left(\operatorname{Tr}, l_{1}, 0\right) \in s_{1}$, then (by definition of truth) $l_{1}$ would be true, contradicting Bivalence. In this sense, we can say that $\left(T r, l_{1}, 0\right)$ diagonalizes out of situation $s_{1}$. Assertive accessible liars are false propositions, but the facts that express about these propositions that they are false ones diagonalize out of the actual situations they are about. Summing up: $l_{1}$ is about an actual situation $s_{1}, l_{1}$ says of $s_{1}$ that it is of the type in which $l_{1}$ is false, $l_{1}$ is false, the falsity of $l_{1}$ is a fact of the actual world, but it is not a fact of situation $s_{1}$.

Consider now an asserted inaccessible liar determined by the non actual situation $s_{2}: l_{2}=\left\{s_{2},\left[\left(T r, l_{2}, 0\right)\right]\right\}$. In this case, the proposition can be either true or false. Suppose $l_{2}$ is true. That would make $\left(\operatorname{Tr}, l_{2}, 0\right) \in s_{2}$, but that does not make $l_{2}$ false, because $s_{2}$ is not a part of total model $\mathfrak{A}$. Suppose now that $l_{2}$ is false. Thus, $\left(\operatorname{Tr}, l_{2}, 0\right) \notin s_{2}$ (by definition 9). Now, by 12 , if $l_{2}$ is false, $\left(\operatorname{Tr}, l_{2}, 0\right)$ is in total model $\mathfrak{A}$. Again, no problem. Except for the fact that falsity of asserted liars diagonalize out of the situation they are about, we have no restrictions here. Nevertheless, we can say that if $s_{2}$ is a situation that includes the fact that $l_{2}$ is false, then the asserted inaccessible liar is true.

Theorem 14. Let $l_{2}=\left\{s_{2},\left[\left(T r, l_{2}, 0\right)\right]\right\}$, where $s_{2}$ is non actual, but $\left(T r, l_{2}, 0\right) \in s_{2}$. Thus $l_{2}$ is a true proposition.

Proof. Suppose (for reductio) that $l_{2}$ is false. By definition of truth, $\left(\operatorname{Tr}, l_{2}, 0\right) \notin s_{2}$, contradicting hypothesis. Therefore, Bivalence implies that $l_{2}$ is true.

Theorem 15. Denied accessible liars are true propositions.

Proof. Let $d_{1}=\neg\left\{s_{1},\left[\left(\operatorname{Tr}, d_{1}, 1\right)\right]\right\}$, where $s_{1}$ is actual. Suppose (for reductio) that $d_{1}$ is false. By definitions 9 and $11,\left(T r, d_{1}, 1\right) \in s_{1}$. Hence, $\left(\operatorname{Tr}, d_{1}, 1\right)$ is in $\mathfrak{A}$ (a total model), because $s_{1}$ is actual. This would make (by 12) $d_{1}$ true, contradicting Bivalence. Therefore, by Bivalence again, $d_{1}$ must be true.

Notice that analogously to what happens with the falsity of asserted accessible liars, the fact that denied accessible liars are true also diagonalizes out of the situations they are about. We proved above that $d_{1}$ is true and this is fine, because $s_{1} \neq \mathfrak{A}$. 
Otherwise, it would be the case that $\left(\operatorname{Tr}, d_{1}, 1\right) \in s_{1}$, making $d_{1}$ false. Thus, $\left(\operatorname{Tr}, d_{1}, 1\right)$ can be a fact of the actual world, but it needs to be out of situation $s_{1}$ itself.

Concerning denied inaccessible liars, we can say something similar to what has been said about asserted inaccessible ones. Let $d_{1}$ be as above, but now suppose $s_{1}$ is non actual. We can accept that $d_{1}$ is true. In this case, $\left(\operatorname{Tr}, d_{1}, 1\right)$ is in the total model of the world $\mathfrak{A}$, but it can not be in situation $s_{1}$ itself. That is ok, because the situation $s_{1}$ is not even a proper part of $\mathfrak{A}$. We can also accept that $d_{1}$ is false. In this case, $\left(\operatorname{Tr}, d_{1}, 1\right) \in s_{1}$ and $\left(\operatorname{Tr}, d_{1}, 0\right)$ is in the total model $\mathfrak{A}$. No paradox, because $s_{1}$ is non actual.

At this point, the reader can be a little bit upset about inaccessible propositions and non actual situations. Maybe it is not so clear what an inaccessible proposition is supposed to be. Nevertheless, we can simplify what can be said about the liars, leaving aside non actual situations and inaccessible propositions. Let's just say that simple liar sentences (like sentence (2) of this paper) ambiguously give rise to at least two different accessible propositions determined by the two schema: $l_{1}$ and $d_{1}$. The first, $l_{1}$, is the one that says of a given actual situation $s_{1}$ that it is of the type in which $l_{1}$ is false (not true). The second, $d_{1}$, is the one that says of a given actual situation $s_{1}$ that it is not of the type in which $d_{1}$ is true. The first proposition is false, the second one is true and none of them give rise to paradox. This is how Situation Theory deals with the so called simple liars.

Next we briefly show that the same framework also allows us to deal with different versions of the liar. For simplicity we are going to suppose that situation $s$ is actual and deals only with the asserted version of propositions. As in the case of simple liars, denied and inaccessible versions also preserve classical interpretations. Actually, we are going to talk just about two different and more challenging versions of the Liar, Curry's Paradox and Yablo's Paradox. We believe that this shall be enough.

We begin with Curry's Paradox. Consider the proposition $c=\{s,[\bigvee X]\}$, where $X=\{[(\overline{\operatorname{Tr}, c, 1})],[(\operatorname{Has}, \operatorname{Max}, 3 \boldsymbol{\phi}, 1)]\}$. Thus $c$ is the proposition that says of situation $s$ that it is of the type in which either $c$ is false or Max has the $3 \mathbf{c}$. This is a disjunctive way of representing a conditional situation type, the one in which if $c$ is true, then Max has the 3h. Remember that, as discussed in the first section, this proposition behaves more like a contingent version of the liar. Curry's version occurs by the fact that we could replace second disjunct for anything, making everything true. Here we are going to consider two possibilities: i) situation $s$ verifies the fact that Max does have the 3 and ii) situation $s$ doesn't verify that Max has the 3. Again, no paradox.

Theorem 16. Let $c$ as defined above and (Has, Max, 3\$, 1) $\notin s$. Hence, $c$ is false.

Proof. Suppose (for reductio) that $c$ is true. Thus either $c_{1}=\{s,[(\overline{\operatorname{Tr}, c, 1})]\}$ is true or $c_{2}=\{s,[(\operatorname{Has}, \operatorname{Max}, 3 \boldsymbol{\uparrow}, 1)]\}$ is true. By hypothesis, $c_{2}$ is false. Hence $c_{1}$ is true and 
$(\operatorname{Tr}, c, 0) \in s$. Nevertheless, $s$ is (by hypothesis) actual, then $c$ would be false (by 12), contradicting Bivalence. Therefore, $c$ has to be false.

Notice that from the fact that $c$ is false no paradox follows. It follows that $c_{1}$ and $c_{2}$ are both false, that means, $(\operatorname{Tr}, c, 0) \notin s$ and (Has, Max, $\left.3 \mathbf{p}, 1\right) \notin s$, but this is fine because the last consequence agrees with hypothesis and $s \neq \mathfrak{A}$. Otherwise, we would be in trouble here, because falsity of $c$ also implies that $(\operatorname{Tr}, c, 0)$ is in the total and actual model of the world $\mathfrak{A}$.

Theorem 17. Let $c$ as defined above and (Has, Max, 3\$, 1) $\in s$. Hence, $c$ is true.

Proof. Suppose (for reductio) that $c$ is false. It follows that both $c_{1}$ and $c_{2}$ are false. This would imply that (Has, Max , 3\$, 1) $\notin s$, contradicting hypothesis. By Bivalence, $c$ is true.

Let's now take Yablo's Paradox into account. In the first section, it was expressed by an infinite sequence of sentences, $\left(7_{1}\right),\left(7_{2}\right), \ldots\left(7_{n}\right),\left(7_{n+1}\right), \ldots$, such that each one of them said, about all sentences after itself in the sequence, that they were all not true. In the new setting, Yablo's propositions would be given by the following schema:

$$
\begin{aligned}
& \left(Y_{1}\right)\left\{s,\left[\bigwedge X_{1}\right]\right\} \\
& \left(Y_{2}\right)\left\{s,\left[\bigwedge X_{2}\right]\right\} \\
& \quad \vdots \\
& \left(Y_{n}\right)\left\{s,\left[\bigwedge X_{n}\right]\right\} \\
& \left(Y_{n+1}\right)\left\{s,\left[\bigwedge X_{n+1}\right]\right\}
\end{aligned}
$$

Where $X_{1}=\left\{\left[\left(\operatorname{Tr}, Y_{2}, 0\right)\right],\left[\left(\operatorname{Tr}, Y_{3}, 0\right)\right], \ldots\right\}, X_{2}=\left\{\left[\left(\operatorname{Tr}, Y_{3}, 0\right)\right],\left[\left(\operatorname{Tr}, Y_{4}, 0\right)\right], \ldots\right\}$ and so on. Thus, for each proposition $Y_{x}$ in the sequence above, it says of situation $s$ that it is of the type in which $\left(\operatorname{Tr}, Y_{y}, 0\right)$ verifies, for all $y>x$. Notice that $Y_{m}$ does not say that $Y_{m+1}$ is false, but says that $\left(\operatorname{Tr}, Y_{m+1}, 0\right) \in s$. Of course, given that $s$ is actual, if $Y_{m}$ is true, $Y_{m+1}$ would have to be false. Nevertheless, this difference is strong enough to block the old paradox.

Theorem 18. All propositions in a Yablo sequence are false.

Proof. Let $Y_{m}$ be any proposition in a Yablo sequence. Suppose (for reductio) that $Y_{m}$ is true. This implies that all the following infinite propositions are true: $Y_{m, 1}=$ $\left\{s,\left[\left(\operatorname{Tr}, Y_{m+1}, 0\right)\right]\right\}, Y_{m, 2}=\left\{s,\left[\left(\operatorname{Tr}, Y_{m+2}, 0\right)\right]\right\}$, and so on. Hence, we would have that $\left(\operatorname{Tr}, Y_{m+1}, 0\right) \in s,\left(\operatorname{Tr}, Y_{m+2}, 0\right) \in s$, and so on. We take for granted that $s$ is actual, so 
this also implies that $Y_{m+1}$ is false, $Y_{m+2}$ is false, $Y_{m+3}$ is false, and so on. Nevertheless, this is not possible. Suppose, for instance that $Y_{m+1}$ is false. This would imply that at least one of the following conditions hold: $\left(\operatorname{Tr}, Y_{m+2}, 0\right) \notin s,\left(\operatorname{Tr}, Y_{m+3}, 0\right) \notin s$, and so on. If one of these conditions hold, however, $Y_{m}$ is false, contradicting hypothesis. Therefore, by Bivalence, $Y_{m}$ is false, for any positive integer $m$.

Notice that accepting that $Y_{m}$ is false (for any positive integer $m$ ) doesn't imply that one of the following is true or false: $Y_{m+1}, Y_{m+2}, Y_{m+3}$, and so on. It only implies that at least one of the following holds: $\left(\operatorname{Tr}, Y_{m+1}, 0\right) \notin s,\left(T r, Y_{m+2}, 0\right) \notin s$, $\left(\operatorname{Tr}, Y_{m+3}, 0\right) \notin s$ or so on. No problem. Situation $s$ can in fact be almost empty. It is still possible that it is an actual situation and the world is full with all facts about falsity of Yablo's propositions about $s$. This way we can block paradox.

\section{Final Remarks}

As we have been trying to argue for here, Situation Theory is a very good approach to the liar paradoxes. First, it makes it possible to deal with propositions, making room for the role context plays in a very pervasive way. It also includes non-wellfounded propositions, which can be seen as an advantage from the perspective of its expressive power. Second, Situation Theory preserves, in its own way, Bivalence and T-Schema. Despite such a classical setting, old paradoxical propositions end up being consistently evaluated as either true or false (not both) in Situation Theory.

At this point, the reader would be trying to devise a revenge version of liars for Situation Theory. We are not going to say that there is no such thing. Nevertheless, it is possible to show that at least one way for doing this is blocked. The obvious way would be taking for granted something like a universal situation $s_{u}$ that includes all facts of the actual world, that means, $s_{u}=\mathfrak{A}$, a total and actual model of the world. Thus, an asserted accessible liar would give us proposition $l_{u}=\left\{s_{u},\left[\left(\operatorname{Tr}, l_{u}, 0\right)\right]\right\}$. Now, if $l_{u}$ is true, $\left(T r, l_{u}, 0\right) \in s_{u}$, but $s_{u}=\mathfrak{A}$ and so $L_{u}$ is false. Otherwise, if $l_{u}$ is false, $\left(\operatorname{Tr}, l_{u}, 0\right)$ is in $\mathfrak{A}$, but $s_{u}=\mathfrak{A}$ and so $l_{u}$ is true. We've got paradox, once again. Nevertheless, there is no such universal situation $s_{u}$, as already proved in theorem 7 . Therefore, at least this argument for revenge is blocked.

It is possible to believe that theorem 7 is an important loss on the expressive power of the theory, that in natural language we can talk about the whole world in a very pervasive way. This is something that can be taken as a strong objection against Situation Theory. The way we block revenge here is by restricting universal situations. In such theory, we could never express ideas about the whole world itself.

This is classically taken to be the main challenge for contextualist views. We are not pretending to give an answer to this in here. All the efforts of this paper have centered on just trying to make clear the very idea behind contextualist views and 
the framework given by Situation Theory. Nevertheless, we advance next some ideas we believe to be in favor of Situation Theory.

First, it is maybe relevant to notice that universal sets are also usually excluded from set theories. If we accept that there is a universal set $U=\{x: x=x\}$, for instance, in $Z F C$, then we could easily show that $Z F C$ is inconsistent. In naive conception of set, we agree that any property $P(x)$ gives rise to a set $A=\{x: P(x)\}$, this is usually called the Naive Schema of Comprehension. Russell's paradox shows that naive conception is inconsistent. We would have to accept the existence of a set like $R=\{x: x \notin x\}$. However, if there is set $R$, then $R \in R$ iff $R \notin R$.

In $Z F C$, the naive comprehension gives place to the Axiom Schema of Separation, which says that any property $P(x)$ and set $B$ give rise to a set $A=\{x \in B: P(x)\}$. Now, if there is a set like $U$ in $Z F C$, we also could prove that there is a set like $R$ in it. As told before, we can change $Z F C$ in some other aspects, like allowing the existence of nonwell-founded sets. Nevertheless, separation seems to be a very important and basic idea. According to it, we make sure that every time we get some set from properties expressed in the language of the theory, it is in fact a subset of some other set already proved to exist. This way we never generate paradoxes like the old ones. Universality is, from a set theoretical and classical. ${ }^{30}$ perspective, an inconsistent idea ${ }^{31}$

Universal sets are not supposed to exist, even if we put Russell's Paradox aside. Consider, for instance, Cantor's Theorem, according to which, the power set of any set $A$ (whether infinite or not) always has strictly higher cardinal than the set itself, that means, $|A|<\left|A^{*}\right|$. If there is a set like $U$, however, $U^{*}$ would be also a subset of $U$ (because $U^{*}$ would also be a set of sets). But how could we make sense of $|U|<\left|U^{*}\right|$ if everything inside $U^{*}$ is also in $U$ ?

Some could say that the very idea of a universal situation makes no sense. A universal situation would be one determined by the perspective of an omniscient agent in a universal space-time location. This is a very strange kind of agent and also a very strange kind of location. They would have to be distinguished from all agents and all locations inside proper parts of the world itself. In a way, they are not in the world, but over the world itself. Maybe these ideas make sense in a religious or metaphysical conception of the world, but this is surely not common ground at this point. Therefore, we could leave to universality defenders the task to explain what they understand by the notions of universal agent and universal location.

Situation Theory is a bottom up theory of the world. We begin with the notions of agents, space-time locations and relations, then we go on through SOA's, situations, propositions and models of the world. Universal locations and agents, if they really make any sense, are supposed to be explained in a top down theory. Therefore, we are not forced here to make sense of these notions. In fact, we could actually be happy that we don't have to deal with all different philosophical problems that these notions carry with themselves. 
Anyway, by blocking universal situations, we can do the trick of solving the liars with no revenge. For now this is maybe just a trick, but one such that gives us good ideas about the paradoxes.

\section{References}

Barwise, J.; Etchemendy, J. 1987. The Liar: an essay on truth and circularity. New York: Oxford.

Barwise, J.; Perry, J. 1983. Situations and Atittudes. Massachusetts: MIT Press.

Burge, T. 1979. Semantical paradox. Journal of Philosophy 76: 169-198.

Glanzberg, M. 2001. The liar in context. Philosophical Studies 103(3): 217-251.

- 2004. A contextual-hierarchical approach to truth and the liar paradox. Journal of Philosophical Logic 33(1): 27-88.

Kripke, S. 1975. Outline of a theory of truth. Journal of Philosophy 72: 690-716.

Priest, G. 1984. Logic of paradox revisited. Journal of Philosophical Logic 13: 153-179.

- 2006. In Contradiction. Oxford: Oxford University Press.

Russell, B. 1908. Mathematical logic as based on the theory of types. American Journal of Mathematics 30: 222-262.

Tarski, A. 1935. O conceito de verdade nas linguagens formalizadas. In: Tarski (2006), pp.19148.

—. 1944. A concepção semântica da verdade e os fundamentos da semântica. In: Tarski (2006), pp.157-201.

- 1969. Verdade e demonstração. In: Tarski (2006), pp.203-233.

- 2006. A Concepção Semântica da Verdade - Textos Clássicos de Tarski. Organização de Cezar Mortari e Luiz Henrique Dutra. São Paulo: Unesp.

van Fraassen, B. C. 1968. Pressuposition, implication and self-reference. Journal of Philosophy 65(5): 136-152.

Yablo, S. 1993. Paradox whithout self-reference. Analysis 53-4: 251-252.

\section{Notes}

${ }^{1}$ Sometimes it might seem reasonable to distinguish between 'not true' and 'false'. This distinction could allow someone to say that 1 isn't true whithout the paradoxical consequence that it is also true. Of course, such a distinction would not help very much, because then (2) would be just a strenghtened version of (1). For that reason, we here assume that a proposition is not true iff it is false.

2 By 'valid arguments' we mean the classically valid ones. From a semantical perspective of Classical Logic, we assume Bivalence and classical interpretations of logical symbols. It follows from this the following very important results: For any formula $F(x)$, any sentences $A, B$ and any set of sentences $\Gamma$, i) Double Negation (DN): $\Gamma \vDash A$ iff $\Gamma \vDash \neg \neg A$. ii) Excluded Middle (EM): $\Gamma \vDash A$ or $\Gamma \vDash \neg A$. iii) Non-Contradiction (NC): $\Gamma \not \models A$ or $\Gamma \not \models \neg A$. iv) Explosion (E): if $\Gamma \vDash A \wedge \neg A$, then $\Gamma \vDash B$. v) Modus Ponens (MP): if $\Gamma \vDash A \rightarrow B$ and $\Gamma \vDash A$, then $\Gamma \vDash B$. vi) Contraction (C): if $\Gamma \vDash A \rightarrow(A \rightarrow B)$, then $\Gamma \vDash A \rightarrow B$. vii) Leibniz Law (LL): $\Gamma \vDash t_{1}=t_{2}$ 
iff $\Gamma \vDash F\left(t_{1}\right) \leftrightarrow F\left(t_{2}\right)$. Of course, this is not everything we could emphasize about classical logic, but it would be enough for the purposes of this paper.

${ }^{3}$ We are taking here a propositional version of Bivalence. Usually, Bivalence doesn't mention propositions (it takes sentences as the truth bearers).

${ }^{4}$ The one expressed by the predicate 'true' of English, for instance.

${ }^{5}$ Where $\operatorname{Tr}(p)$ names the proposition that says of $p$ it is true. Usually, T-Schema is not presented in propositional form. The sentencial version of it comes from Tarski's work as Convention T (Tarski 1935; 1944; 1969). For Tarski a materially adequate definition of truth should imply all instances of such schema.

${ }^{6}$ In its stronger version, Diagonal Lemma says that if $\mathscr{L}$ is an interpreted first order language capable of expressing its very own syntax, then, for any formula $A(x)$ of $\mathscr{L}$ (where $x$ is the only free variable in $A$ ), there is a term $\lambda$ of $\mathscr{L}$ such that $\lambda=\ulcorner A(\lambda)\urcorner$ (where $\ulcorner A(\lambda)\urcorner$ is a term that names $A(\lambda)$ ). This is self-reference. An expression that says of itself that it satisfies $A(x)$. The lemma also applies for formulas with more then just one free variable and that is exactly how we can use it to formalize cases like those of (3), (4) and (7) above.

${ }^{7}$ The reader can check, for instance, that $\in$ is non-well-founded on $\Omega=\{\Omega\}, \Gamma=\{\Delta\}$ and $\Delta=\{\Gamma\}$, but $\in$ is also non-well-founded on the following infinite list of sets: $\Theta_{1}=$ $\left\{\Theta_{2}\right\}, \Theta_{2}=\left\{\Theta_{3}\right\}, \Theta_{3}=\left\{\Theta_{4}\right\},(\cdots)$. We can easily see some structural similarities between non-well-founded sets like these and sentences (1)-(7).

${ }^{8}$ Usually reference is taken as a relation between agents and objects. Hence, it makes no sense to say that (1) refers to a given sentence or proposition, but someone could use (1) to refer to a sentence or a proposition. We are taking reference to be a relation between sentences or propositions and anything else. This is not accurate, but it's not wrong either. It's just a short way we take for the purposes of the current investigation.

${ }^{9}$ In what follows, we are supressing explicit use of T-Schema for simplicity.

10 This fancy version of liars was first designed by Yablo in (1993).

${ }^{11}$ A good example of this is the work of Burge in (1979). Burge actually mixes hierarchies of levels of truth with truth value gaps and contextualism. Hence, his approach is actually in the intersection between hierarchical views, non-classical views and contextualist views.

${ }^{12}$ Russell's Theory of Types presented in Russell (1908) is an example of something like this.

${ }^{13}$ Consider the function $\mathrm{T}: P^{*} \rightarrow P^{*}$ (where $P^{*}$ is the set of all subsets of propositions of some given language). $\mathbf{T}\left(\Gamma_{m}\right)$ takes for input the set of all true propositions in level $m$ and gives for output the set of all propositions that come out true in level $m+1$ (say $\Gamma_{m+1}$ ), after using the first set as the extension of $T r_{m}$. Thus, $\Gamma_{\omega}$ is a fixed point of $\mathbf{T}$ iff $\mathbf{T}\left(\Gamma_{\omega}\right)=$ $\Gamma_{\omega}$. Kripke's hierarchies (in 1975) have fixed points, but it also mixes hierarchies with nonclassical interpretations.

14 Outlined in Kripke (1975).

15 We can also take Van Fraassen's work (in van Fraassen, 1968) as an example of the Gap View. They both deal with sentences, but we can ignore this here, because the revenge arguments we'll be showing next could be presented in sentential form too.

${ }^{16}$ Kripke follows the strawsonian position about cases of reference failing. See footnote 16 in (1975).

17 Like Priest (1984; 2006).

${ }^{18}$ In ZFC we have Axiom of Extensionality, which says: $\Gamma=\Delta$ iff $\forall x(x \in \Gamma \leftrightarrow x \in \Delta)$, for all sets $\Gamma$ and $\Delta$. 
${ }^{19}$ We can find the general idea behind Contextualist View in Burge (1979), Barwise and Etchemendy (1987), Glanzberg (2001; 2004).

${ }^{20}$ We are going to fix the actual world, for simplicity, even when talking later about models of worlds.

${ }^{21}$ Notice that we could be more precise on the space-time location, but this would only determinate a more specific and precise situation.

${ }^{22}$ In Barwise and Perry (1983), the concept of attunement is more cautiously taken into account.

${ }^{23}$ In Barwise and Etchemendy (1987), authors present a language in formal way before talking about situations and propositions. The main problem is that, by doing that, they have also to be very economic about what counts for objects and relations there. We are taking the theory in an informal way, but let $\mathscr{A}$ be a class of atoms from where we take objects $o_{1}, o_{2}, o_{3}, \ldots$ and relations $R_{1}, R_{2}, R_{3}, \ldots$ We are not considering the required restrictions on $\mathscr{A}$ that would be necessary.

${ }^{24}$ Here, $\operatorname{Tr}$ is the one place relation of a proposition being true and $p$ is any proposition.

${ }^{25}$ Here, we assume that we have all classes as available objects inside the class of atoms $\mathscr{A}$.

${ }^{26}$ A class that includes all sets cannot itself be a set, on the risk of retrieving the old set theoretic paradoxes. In $Z F C^{-} / A F A$, the existence of a set $U$ that includes all sets is forbidden. Notice that we can show in $Z F C^{-} / A F A$ that $U$ is not a set, but we can not show it to be a proper class. The notion of proper class is not formalizable in such theory. This makes the very definition of total models given above impossible inside $Z F C^{-} / A F A$. This surely shows we would need a better theory to formalize this approach on the Liar, one such that would extend $Z F C^{-} / A F A$ and would allow us to talk about proper classes. In Barwise and Etchemendy (1987), 7 above is not a theorem. Of course, the fact that we can prove 7 the way we did here doesn't give restrictions on universality a more natural or justified status. It depends on how we define things and the set theory we chose at first. Anyway, we hereby are just presenting the framework of Situation Theory in its best form and this is necessary to block revenge, as we'll see later.

${ }^{27}$ Otherwise, propositions would have to be proper classes.

${ }^{28}$ There is maybe an objection here presented by a blind referee. T-Schema holds only in total models, but as proved before, there is no situation which is a total model, a total model of the world is always a proper class. If this implies that total models doesn't exist, then we have a problem. How could we ever say that total models preserve T-Schema? What we think about that is: Total models exist, they just can not be taken as situations, but proper classes. Models are just artifacts that we use to represent pieces of the world, but there can be a total model of the world, even if it could never be grasped by a real agent in a real piece of the world.

${ }^{29}$ In general, Barwise and Etchemendy (1987) calls accessible a proposition that is about an actual situation and, inaccessible one that is about a non actual situation.

${ }^{30}$ Of course, we could also change Logic here.

${ }^{31}$ Of course, as pointed out by a blind referee, a dialetheist could turn the table against me here and say that, because universality is an important feature to express, we have even more reasons to reject both $Z F C$ and Situation Theory. Nevertheless, as I said before, I'm not pretending to have arguments against universalism, but just showing that universalities also carry their own problems. 